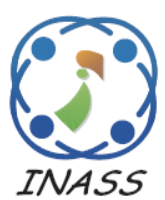

\title{
A Comparison of Machine Learning and Deep Learning Methods with Rule Based Features for Mixed Emotion Analysis
}

\author{
Christy Daniel ${ }^{1 *}$ \\ Shyamala Loganathan ${ }^{1}$ \\ ${ }^{1}$ School of Computer Science and Engineering, VIT University, Chennai, India \\ * Corresponding author's Email: dchristy.daniel2014@vit.ac.in
}

\begin{abstract}
Multi-class classification of sentiments from text data still remains a challenging task to detect the sentiments hidden behind the sentences because of the probable existence of multiple meanings for some of the texts in the dataset. To overcome this, the proposed rule based modified Convolutional neural network-Global Vectors (RCNN-GloVe) and rule-based modified Support Vector Machine - Global Vectors (RSVM-GloVe) were developed for classifying the twitter complex sentences at twelve different levels focusing on mixed emotions by targeting abstract nouns and adjective emotion words. To execute this, three proposed algorithms were developed such as the optimized abstract noun algorithm (OABNA) to identify the abstract noun emotion words, optimized complex sentences algorithm (OCSA) to extract all the complex sentences in a tweet precisely and adjective searching algorithm (ADJSA) to retrieve all the sentences with adjectives. The results of this study indicates that our proposed RCNNGloVe method used in the sentiment analysis was able to classify the mixed emotions accurately from the twitter dataset with the highest accuracy level of $92.02 \%$ in abstract nouns and $88.93 \%$ in adjectives. It is distinctly evident from the research that the proposed deep learning model (RCNN-GloVe) had an edge over the machine learning model (RSVM-GloVe).
\end{abstract}

Keywords: Deep learning, Support vector machine, Fuzzy rules, Natural language processing, Word embedding, Convolutional neural network.

\section{Introduction}

In this era of prominent social networking, people are often sharing and expressing their feelings, emotions and opinions to the world through social networking sites such as Facebook, Twitter, Snapchat, Instagram, and Tumbler [1-4]. Among this array of social networking applications, Twitter, which is widely considered as one of the most popular social media networks, has huge amount of social interactions happening every day. Statista reports that over 500 million tweets being shared everyday across the globe [5]. The number of such interactions has always been in a rising trend with more and more new interactions and users joining this platform as days pass by. These tweets are often expressions of human emotions or opinions or strong feelings of the situation that occurs in people's personal lives or in society. Emotions are sometimes considered as mixed feelings whenever there are combinations of two opposite valence being stated in the same sentence. In other words, mixed emotions are also defined as affective experiences characterized by the co-activation of two emotions, usually opposite in valence [6]. Mixed emotions are focused on both the positive affect, as well as the negative affect which express the two opposite affect about the same event which can be clearly understood by the presence of opposite valences such as happy and sad [6-8], fear and happiness [9], disgust and amusement [10] and hope and fear [11], pleasure and displeasure [10], etc. An example of mixed emotion sentence is "It is extremely a joy to go to Germany, but I am very sad to leave my family". Determining whether a sentence has both positive and negative emotions is an essential part of sentiment analysis (SA) to know people's minds [12]. In our observation, the positive 
and negative valances in mixed emotion sentences are generally of the following two types:

i. Combination of an abstract noun (happiness / sadness) with verb (like / hate) along with their cooccurrence words.

ii. Combination of an adjective (happy / sad) with verb along with their co-occurrence words.

Normally, an abstract noun expresses the strongest emotion that cannot be felt by human senses $[13,14]$, whereas, an adjective is a description of the noun (person / place / object) which also expresses the human's emotion to some extent [15]. This aspect triggered us thought to evaluate the emotions in the sentences focusing on the abstract noun and the adjective. Previously, a lot of existing research works have followed the Pang et al., [16] methods using machine learning (ML) techniques for twitter sentiment analysis. Currently, as deep learning (DL) is gaining momentum in natural language processing (NLP) due to its automatic feature extraction facility, and its efficiency in analyzing complex and huge datasets, our research focus was inclined towards this technique in addition to the existing ML techniques. Convolutional neural network (CNN) is one of the DL techniques which are mainly used for image related research works. But, recently, $\mathrm{CNN}$ is also being applied in NLP research works which can enhance the accuracy of the sentiment classification when compared to ML techniques like support vector machines (SVM), linear regression, etc., Moreover, DL also has the advantage of doing the feature extraction and classification jobs as a single task, when compared to ML. Our contributions for this paper are listed as below:

i. Creation of complex sentence database by using our proposed optimized complex sentences algorithm (OCSA)

ii. Extraction of target sentences (abstract noun, adjective emotion word) from the complex sentence database by using the proposed optimized abstract noun algorithm (OABNA) and adjective searching algorithm (ADJSA) respectively.

iii. Modification of the CNN and SVM by using the twelve fuzzy rules to extract the mixed emotion sentences from the target sentences database.

iv. Classification of the extracted mixed emotion sentences into multi-classes by using our proposed twelve fuzzy rules patterns.

v. Evaluation of extracted mixed emotion sentences by using our proposed models such as rulebased modified Convolutional neural networkGlobal Vectors (RCNN- GloVe) and rule-based modified Support Vector Machine - Global Vectors (RSVM-GloVe).
The remainder of this research paper has been presented under different sections. Section 2 reports on the related works in this area. Section 3 explains the research methods, while, section 4 captures the results and its discussions. Finally, section 5 concludes this research work by throwing light on the further scope for this study.

\section{Review of literature}

In recent times SA has emerged as an active research area for extracting people's thoughts opinions, feelings and behaviours from the user's text data using NLP methods [17].

Adjectives, nouns and adverbs contribute mainly to the SA [15]. In this study on SA, the researchers have tested all the different types of POS tags and finally concluded that the abstract nouns express strong emotions. In this paper, the abstract nouns were classified into positive (happiness, joyfulness, pleasure, etc.) and negative (fear, hate, worry, etc.) abstract nouns.

In the SA model developed by Loia and Senatore [18], Minsky's conception was used by the researchers for finding the emotions and was modelled as a fuzzy set. The fuzzy modifier was used in this research for tuning the emotions and the study focused only on the abstract nouns present in the simple sentences using Plutchik's flower model [19] for SA. However, this model could not able to perform well for the negative polarities.

Most of the research work on sentiment classification using twitter dataset can be categorized with respect to the used supervised methods [20], based on training through linear classifiers like Random Forest, Naïve Byes, logistic regression, Maximum Entropy and Support Vector Machine utilizing feature mixtures like a bag of words, Word $\mathrm{N}$-grams, Parts of Speech, etc [21]. In another research [22] the researchers have used ML algorithms to predict the trends in the stock market by using SVM and Random Forest Model. SVM algorithms were used in most of the previous works on aspect level SA [23]. The majority of the SA works in Arabic language texts have used lexicons or basic ML methods [24]. In research for comparing different basic ML techniques, the researchers have built Multinomial Naive Bayes, SVM classifiers, and Char-level models. The results of this research have proved that SVM has performed better when compared with other models [25]. In another research, the researchers have demonstrated that a hybrid approach, with the combination of two ML approaches using SVM and the semantic orientation proving effective for SA [26]. 
The experimental results of research [27] where the researchers have built a new model called global Vector - Deep Convolutional Network have shown that the implementation of the binary task for classifying the tweet data into positive or negative sentiments proved a better approach. In research [3] for improving the understanding of the sentiments, to reduce the loss of local information and to capture the long-term dependencies, the researchers have combined the recurrent neural networks (RNN) with CNN. Through this approach, the researchers obtained better accuracy on several benchmarked datasets. In a study [28] where a framework developed by jointly combining CNN and RNN on top of unsupervised and pre-trained word vectors performed well and achieved better classification accuracy when compared with several other methods. In a multi-class SA research [29], the researchers have used an open-source tool named SENTA to extract the features for SA, where, they classified a set of tweets into 7 different classes. This multi-class SA obtained an accuracy of 60.2 percent for the twitter dataset.

From the array of the literature surveyed it was observed that there was limited or no SA research focusing on mixed emotions from complex sentences targeting abstract nouns and adjectives. It was also found from our literature review that there are no indepth research works on modified models of CNN using fuzzy rules for complex sentences. Eventually, this has paved way for our study on proposed modified RCNN and modified RSVM.

\section{Materials and methods}

The overall architecture of the proposed RCNN and RSVM with the GloVe word embedding model is presented in Fig. 1. The twitter dataset from the sentiment140 project, introduced by Go et al., [30] was used for this research. Later, the Natural Language Tool Kit [31] was used to pre-process the twitter dataset by converting the unstructured data into structured data. The pre-processing steps are as follows: conversion of the abbreviations to their original words, the removal of symbols, unwanted characters, words of foreign languages (languages other than English), URLs and performing the tokenization finally. Then the pre-processed data got assigned with the part-of-speech tag to each word by using Stanford POS tagger. After the POS tagging, a complex sentence database was created by applying our proposed OCSA to the tagged data. From this

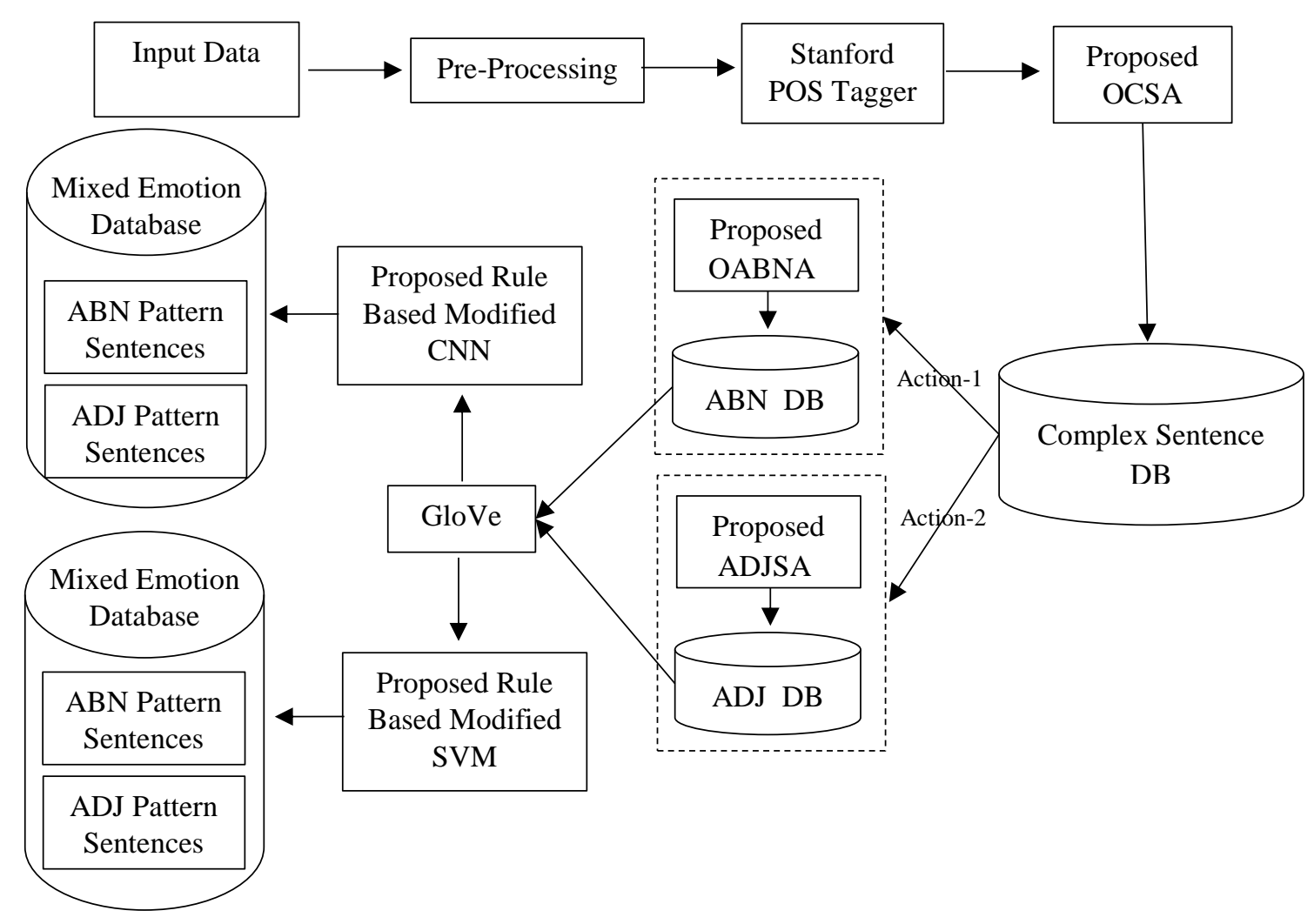

Figure. 1 Architecture of the proposed RCNN and RSVM with GloVe word embedding 
newly created complex sentence database, the target sentences such as abstract noun complex sentences and adjective complex sentences were extracted by applying the proposed OABNA and ADJSA respectively.

In the case of extracting the abstract noun complex sentences, a manually created abstract noun database was used for verifying the identified abstract noun words extracted by the OABNA through mapping, as there was no existing abstract noun database available. Whereas, similar mapping for verifying the accuracy of extracted adjective complex sentences was not exclusively performed as the adjectives in the complex sentences get distinctly tagged by the Stanford POS tagger itself. Then the extracted complex sentences of abstract nouns and adjectives were converted into vector values by using Global Vectors (GloVe). Finally, the vectorized data were subjected to our proposed modified RCNN and RSVM for the evaluation of mixed emotions.

\subsection{Dataset}

For the experiment purposes, the Sentiment140twitter dataset [32] consists of $1,600,000$ sentences, created by the graduate students of Stanford University was used. Several academic papers and research works have been conducted using this dataset. As this dataset is very huge, our research focused on only 400,000 random sentences from $1,600,000$ sentences. Later, to cleanse the data preprocessing was done on the randomly selected 400,000 sentences, then the proposed OCSA got applied to it and finally 121,000 complex (sentences with conjunctions) sentences were extracted. From this, 45,356 complex sentences with abstract nouns and 70,361 complex sentences with adjectives were obtained and subjected to further classifications by using rule-based modified CNN and SVM. Other remaining sentences were ignored. Training and testing ratios followed were $80 \%$ and $20 \%$ respectively.

\subsection{Proposed optimized complex sentences algorithm (OCSA)}

The complex sentences are sentences with conjunctions (CC). The proposed algorithm to identify the complex sentences (OCSA) can be seen in Fig. 2. Initially, in line number 2, the proposed OCSA searches the CC POS tag in the twitter sentences by leaving all the simple sentences. In line number 3 , the complex sentences with multiple numbers of $\mathrm{CC}$ were also considered by the OCSA algorithm for the complex sentence database. In line number 3, it searches the conjunction (CC) and also

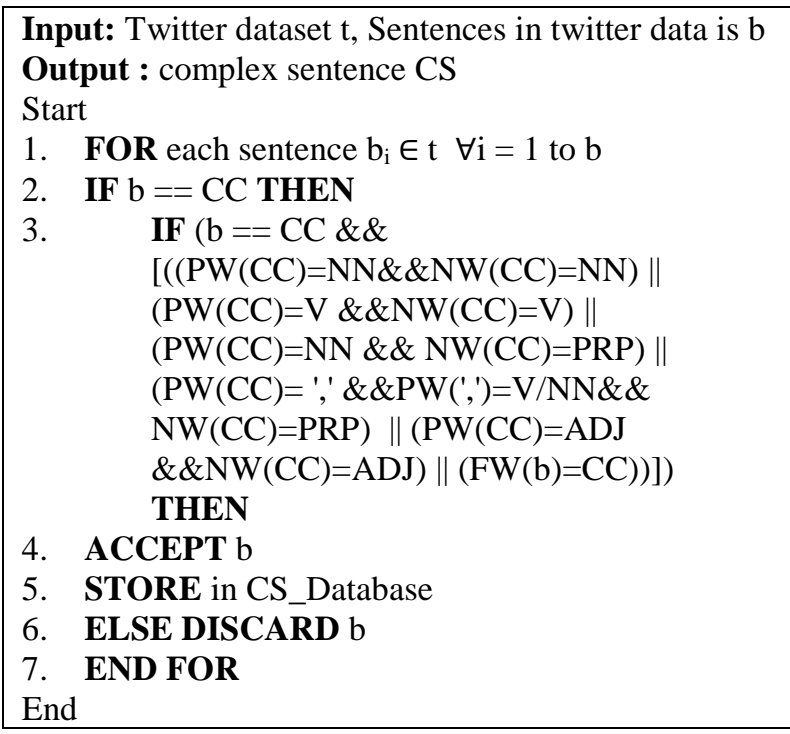

Figure. 2 Optimized complex sentences algorithm (OCSA)

Table 1. List of the conjunctions

\begin{tabular}{|l|l|}
\hline $\begin{array}{l}\text { Types of } \\
\text { Conjunction }\end{array}$ & Examples \\
\hline 1. Coordinating & For, and, nor, but, or, yet, so, ',' \\
\hline 2. Subordinating & $\begin{array}{l}\text { Cause \& Effect: Because, } \\
\text { since, as } \\
\text { Time: When, before, after, } \\
\text { once, until, whenever, since, } \\
\text { while } \\
\text { Place: Place, wherever } \\
\text { Condition: If, unless, in case } \\
\text { Contrast: Although, though, } \\
\text { whereas }\end{array}$ \\
\hline 3. Correlative & $\begin{array}{l}\text { Either...or, neither...nor, not } \\
\text { only...but also, both...and }\end{array}$ \\
\hline
\end{tabular}

searches the multiple $\mathrm{CC}$ with all the possibilities, which is given in line 3 , such as, the previous word and the next word of conjunction having nouns (happiness and joy), etc., This is because, our target word may occur in that position and sometimes, otherwise, there were possibilities for not considering those target complex sentences for our analysis.

The lists of conjunctions are presented in the Table 1 and the Table 2 elaborates the notations used in this paper in a detailed manner.

\subsection{Proposed optimized abstract noun algorithm (OABNA)}

The proposed algorithm (OABNA) for identifying the abstract noun words based on the location of the word's POS tag from the complex sentence database is presented in Fig. 3. Once, the extracted complex sentences are fed into the optimized abstract noun algorithm as input, it checks whether the previous word of conjunction and the last 
Table 2. List of the notations

\begin{tabular}{|c|c|c|}
\hline S.No & Notations & Description \\
\hline 1. & $\mathrm{t}$ & Tweet Dataset \\
\hline 2. & $\mathrm{~b}$ & Twitter dataset sentences \\
\hline 3. & $\mathrm{CS}$ & Complex Sentence \\
\hline 4. & DB & Database \\
\hline 5. & LW & Last Word \\
\hline 6. & NW & Next Word \\
\hline 7. & $\mathrm{NN}$ & Noun Singular \\
\hline 8. & NNS & Noun Plural \\
\hline 9. & NNP & Proper Noun singular \\
\hline 10. & NNPS & Proper Noun Plural \\
\hline 11. & Det & Determiner \\
\hline 12. & ADJ & Adjective \\
\hline 13. & Adv & Adverb \\
\hline 14. & ADJ_DB & Adjective Database \\
\hline 15 & PW & Previous Word \\
\hline 16 & $\mathrm{CC}$ & Conjunction \\
\hline 17 & PRP & Preposition \\
\hline 18 & $\mathrm{~V}$ & Verb \\
\hline 19 & ABN_DB & Abstract Noun Database \\
\hline 20 & P_ABN & Positive Abstract Noun \\
\hline 21 & N_ABN & Negative Abstract Noun \\
\hline 22 & P_ADJ & Positive Adjective \\
\hline 23 & N_ADJ & Negative Adjective \\
\hline 24 & Co_W & Co-Occurrence Word \\
\hline 25 & RB & Adverb \\
\hline 26 & JJS & Adjective Superlative \\
\hline 27 & JJR & Adjective Comparative \\
\hline 28 & RBS & Adverb Superlative \\
\hline 29 & RBR & Adverb Comparative \\
\hline 30 & $\mathrm{UH}$ & Interjection \\
\hline 31 & P_TRVB & Positive_Transitive Verb \\
\hline 32 & N_TRVB & Negative_Transitive Verb \\
\hline 33 & CS_Database & Complex Sentence Database \\
\hline 34 & FW & First Word \\
\hline 35 & ADJ_DB & Adjective Database \\
\hline 36 & ABN_ME_L1 & $\begin{array}{l}\text { Abstract Noun } \quad \text { Mixed } \\
\text { Emotion Level1 }\end{array}$ \\
\hline 37 & ADJ_ME_L1 & $\begin{array}{l}\text { Adjective Mixed Emotion } \\
\text { Level }\end{array}$ \\
\hline
\end{tabular}

word of the complex sentences are nouns in line number 3. If that is correct or true, then it maps the identified nouns with the abstract noun database in line number 4 . If there is a match, it will extract those sentences as the target sentences. Otherwise, it will ignore those sentences. Then again as the second level of target sentence extraction, in line number 8 , it checks whether any random word in the complex sentences that were fed as input has nouns. Then, it checks whether the previous word of any random word is a determiner or adjective or adverb, in line number 9. Later, in line number 10, it checks whether the next word of any random word has an adjective or noun or preposition. In line number 11 , the algorithm verifies whether the conditions from line 8 to line 10 are true. If it is true, then, it maps identified any random word with the abstract noun database in line number 12. If there is a match, it will extract those sentences as target sentences. Otherwise, the sentences will get ignored.

The example for the CS is: "It was really an irritation to travel on Mumbai roads, but I always enjoyed most of the roadside food". The POS tags of the above-mentioned sentence are explained below.

$\mathbf{I t}(\operatorname{Pronoun}) \quad$ was $(\mathrm{V}) \quad$ really $(\mathrm{Adv}) \quad \mathbf{a n}(\mathrm{Det})$ irritation $(\mathrm{NN}) \quad$ to $(\mathrm{PRP}) \quad \operatorname{travel}(\mathrm{V}) \quad$ on $(\mathrm{PRP})$ Mumbai(NN) $\operatorname{roads}(\mathrm{NN}) \quad \operatorname{but}(\mathrm{CC}) \quad \mathrm{I}($ Pronoun) always(Adv) enjoyed(V) $\operatorname{most}(J J S) \quad$ of(PRP) the(Det) roadside(NN) food $(\mathrm{NN})$.

Input: Complex Sentence CS, twitter dataset t

Output: Extract all the target (abstract noun) sentences Start

1. FOR each sentences $\mathrm{CS}_{\mathrm{i}} \in \mathrm{t} \forall \mathrm{i}=1$ to $\mathrm{CS}$

2. $\mathrm{CC}=$ conjunction, // Conduct the abstract noun searching task on CS //

3. IF the previous word of the $\mathrm{CC}$ or the last word of CS is equal to NNP or NN or NNPS or NNS THEN

4. SEARCH availability of the previous word of CC or last word of CS is in abstract noun database THEN

5. IF $3^{\text {rd }}$ and $4^{\text {th }}$ steps are true THEN

6. ACCEPT CS as the target sentence

7. ELSE DISCARD CS

8. IF any random word from CS is equal to NNP or NN or NNPS or NNS THEN

9. IF the previous word of any random word of CS is equal to Det or ADJ or Adv THEN

10. IF the next word of any random word of CS is equal to ADJ or NN or PRP THEN

11. IF $8^{\text {th }}$ to $10^{\text {th }}$ steps are true THEN

12. SEARCH any random (abstract noun) word is in the abstract noun database THEN

13. ACCEPT CS as the target sentence

14. ELSE DISCARD CS

15. END FOR

End

Figure. 3 Optimized abstract noun algorithm (OABNA) 


\subsection{The proposed adjective searching algorithm (ADJSA)}

The proposed algorithm (ADJSA) to identify and extract the complex sentences with adjectives (ADJ) through the POS tags in the complex sentences is presented in Fig. 4. The extracted CS from OCSA algorithm was fed into the ADJSA for searching the target sentences with an adjective (ADJ) POS tag. Later the searched target sentences get stored in the database, else the data gets discarded.

\subsection{Fuzzy rule based modified CNN}

The proposed RCNN classifies separately the abstract noun and adjective complex sentences from the twitter dataset into twelve different levels. In RCNN, GloVe (Stanford) is global vector for word representation which is used to convert all the words into vectors for word embedding. Let $Q \in R^{s \times|V|}$ is a word vector table generated by GloVe, where $\mathrm{R}$ and $\mathrm{s}$ denote the word vector and vector dimensions. Here, $\mathrm{V}$ indicates a vocabulary size. The new feature vector $v$ is generated when each word from the preprocessed twitter dataset is mapped with the word embedding vector $k_{i} \in R_{S}$ Where, $k$ is each word and $\oplus$ is the concatenation operator. The new feature vector is:

$$
v=k_{1} \oplus k_{2} \oplus k_{3, \ldots \ldots} \oplus k_{n}
$$

A new feature vector gi is produced by the sliding window of length $\mathrm{p}$. Then the input matrix is:

$$
g_{i}=f\left(U \cdot v_{i: i+p-1}+z_{i}\right)
$$

Where, $v_{i: i+p-1}$ is the local vector from $i$ to $i+p-$ $1, U$ and $z_{i}$ are the transition matrix and the bias term respectively, generated within the convolutional for each filter. Here, $f$ is a rectified linear units (ReLu) and activation function in CNN. Next, the computation procedure is started to generate the new feature vector $(g)$ in the convolution section. The new feature vector is as follows:

$$
g=g_{1}, g_{2}, \ldots . g_{s-p-1}
$$

In this work, the K-max pooling is needed to specify the fixed size of the output matrix for the classification which follows after the convolutional layer. Here, a max operation is needed to apply on each resulted filter in the convolutional layer, utilised on the feature vector to increases the speed of the computational tasks.

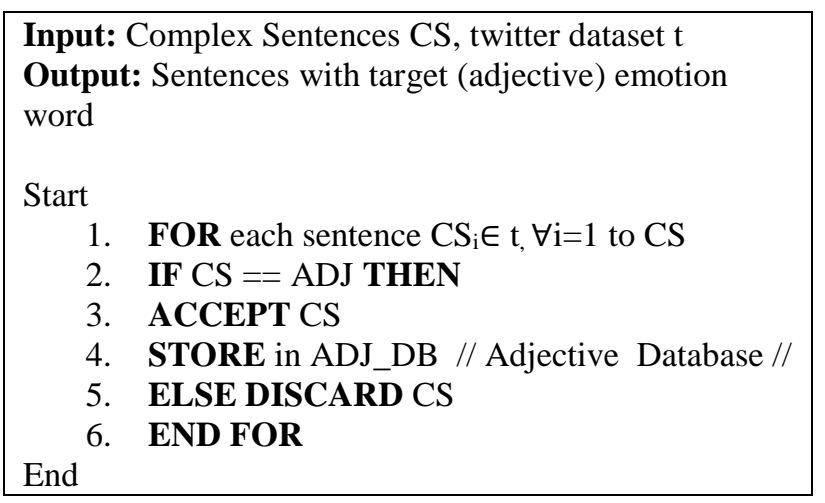

Figure. 4 Adjective searching algorithm (ADJSA)

Now, the K-max pooling was applied to the new feature vector $\mathrm{g}$ on each filter in the convolutional layer. The newly generated output vector $v^{\prime}$ for k-max pooling is as follows:

$$
v^{\prime}=\max \left\{g_{1}, g_{2} \ldots \ldots g_{s-p-1}\right\}
$$

The final layer is the softmax layer which receives feature vector as the input to build the text classifier. The softmax layer classifies the output values into twelve levels of classes for adjective and abstract noun target sentences by using the softmax layer. The softmax function is given below:

$$
p=\operatorname{softmax}\left(U v^{\prime}+z_{i}\right)
$$

Where $p$ is the output vector of the softmax layer. In this layer, the dropout is used during the training period, which helps to regularize the network layers, remove the entire random layer along with its connection and correct its faults from the prior layers.

\subsection{Fuzzy rule based modified SVM}

SVM is a popular ML algorithm which got trained to obtain a word vector by using GloVe word embedding for each sentence in the twitter dataset. Then, the word vector of all the sentences got fed into our proposed model of RSVM-GloVe and trained for classifying the twitter dataset as into twelve levels of abstract noun and adjective set of sentences. In our proposed RSVM-GloVe model, the radial basis function (RBF) kernel has been used for handling the non-linear problems. The features such as bigram, ngram, text vector features, polarity features for sentiment words were applied and experimented on the RSVM-GloVe with RBF kernel.

\subsection{Fuzzy rules}

The fuzzy rules used for this research work are presented in this section below. In our research work, 
the mixed emotion tweet sentences were categorized into twelve levels using fuzzy rules. All the rules listed below (Table 3) were combined with the DL and ML techniques to extract and detect the mixed emotions from the targeted complex sentences of abstract nouns and adjectives. In the rules, the two types of verbs have been used such as transitive and intransitive verbs. A transitive verb is directly connected to the noun (person, object) which tells the human mind more than the intransitive verb, which is not directly connected to the noun. So the transitive verbs are placed before the intransitive verb in the fuzzy rules. The transitive verbs can be identified based on the following patterns (Verb + Noun / (Preposition / Determiner + Noun)) and the remaining is considered as intransitive verbs. Generally, transitive verbs will come along with the modal verbs (can, could, would, must, etc.,) and intransitive verbs will come along with the auxiliary verbs (is, was, did, etc.). Modal verbs usually describe the ability of the person and hence it is placed before the auxiliary verbs along with its cooccurrence words in the fuzzy rules. Among the twelve rules proposed, the first four rules are the mixed emotion patterns without negation words such as Not, No, and Never. The remaining rules from rule 5 to rule 12 are the mixed emotions with the negation words Not, No and Never.

Table 3. Fuzzy rules

\begin{tabular}{|c|c|c|}
\hline No & Rules & Levels \\
\hline 1 & $\begin{array}{l}\text { Target(P_ABN/P_ADJ) } \Lambda \\
\text { Co_W(RB v JJS v JJR v } \\
\text { RBS v RBR v UH) } \Lambda \\
\text { Target(N_TRVB) } \Lambda \\
\text { Co_W(RB v JJS v JJR v } \\
\text { RBS v RBR v UH) }\end{array}$ & $\begin{array}{l}\text { ABN_ME_L1/ } \\
\text { ADJ_ME_L1 }\end{array}$ \\
\hline 2 & $\begin{array}{l}\text { Target(N_ABN/N_ADJ) } \Lambda \\
\text { Co_W(RB v JJS v JJR v } \\
\text { RBS v RBR v UH }) \Lambda \\
\text { Target(P_TRVB }) \Lambda \\
\text { Co_W(RB v JJS v JJR v } \\
\text { RBS v RBR v UH) }\end{array}$ & $\begin{array}{l}\text { ABN_ME_L2/ } \\
\text { ADJ_ME_L2 }\end{array}$ \\
\hline 3 & $\begin{array}{l}\text { Target(P_ABN/P_ADJ) } \Lambda \\
\text { Co_W(RB v JJS v JJR v } \\
\text { RBS v RBR v UH) } \Lambda \\
\text { Target(N_INVB) } \Lambda \\
\text { Co_W(RB v JJS v JJR v } \\
\text { RBS v RBR v UH) }\end{array}$ & $\begin{array}{l}\text { ABN_ME_L3/ } \\
\text { ADJ_ME_L3 }\end{array}$ \\
\hline 4 & $\begin{array}{l}\text { Target(N_ABN/N_ADJ) } \Lambda \\
\text { Co_W(RB v JJS v JJR v } \\
\text { RBS v RBR v UH) } \Lambda \\
\text { Target(P_INVB) } \Lambda \\
\text { Co_W(RB v JJS v JJR v } \\
\text { RBS v RBR v UH) }\end{array}$ & $\begin{array}{l}\text { ABN_ME_L4/ } \\
\text { ADJ_ME_L4 }\end{array}$ \\
\hline 5 & $\begin{array}{l}\text { Target(P_ABN/P_ADJ }) \Lambda \\
\text { Co_W(RB v JJS v JJR v }\end{array}$ & $\begin{array}{l}\text { ABN_ME_L5/ } \\
\text { ADJ_ME_L5 }\end{array}$ \\
\hline
\end{tabular}

\begin{tabular}{|c|c|c|}
\hline & $\begin{array}{l}\text { RBS v RBR v UH) } \Lambda \\
(\text { Modal+not) } \\
\text { Target(N_TRVB) } \Lambda \\
\text { Co_W(RB v JJS v JJR v } \\
\text { RBS v RBR v UH) }\end{array}$ & \\
\hline 6 & $\begin{array}{l}\text { Target(P_ABN/P_ADJ) } \Lambda \\
\text { Co_W(RB v JJS v JJR v } \\
\text { RBS v RBR v UH) } \Lambda \\
(\text { Auxiliary+not) } \\
\text { Target(N_TRVB) } \Lambda \\
\text { Co_W(RB v JJS v JJR v } \\
\text { RBS v RBR v UH) }\end{array}$ & $\begin{array}{l}\text { ABN_ME_L6/ } \\
\text { ADJ_ME_L6 }\end{array}$ \\
\hline 7 & $\begin{array}{l}\text { Target(N_ABN/N_ADJ) } \Lambda \\
\text { Co_W(RB v JJS v JJR v } \\
\text { RBS v RBR v UH) } \Lambda \\
(\text { Modal+not }) \\
\text { Target(P_TRVB }) \Lambda \\
\text { Co_W(RB v JJS v JJR v } \\
\text { RBS v RBR v UH) }\end{array}$ & $\begin{array}{l}\text { ABN_ME_L7/ } \\
\text { ADJ_ME_L7 }\end{array}$ \\
\hline 8 & $\begin{array}{l}\text { Target(N_ABN/N_ADJ) } \Lambda \\
\text { Co_W(RB v JJS v JJR v } \\
\text { RBS v RBR v UH) } \Lambda \\
(\text { Auxiliary+not) } \\
\text { Target(P_TRVB) } \Lambda \\
\text { Co_W(RB v JJS v JJR v } \\
\text { RBS v RBR v UH) }\end{array}$ & $\begin{array}{l}\text { ABN_ME_L8/ } \\
\text { ADJ_ME_L8 }\end{array}$ \\
\hline 9 & $\begin{array}{l}\text { Target(P_ABN/P_ADJ) } \Lambda \\
\text { Co_W(RB v JJS v JJR v } \\
\text { RBS v RBR v UH) } \Lambda \\
(\text { Modal+not) } \\
\text { Target(N_INVB) } \Lambda \\
\text { Co_W(RB v JJS v JJR v } \\
\text { RBS v RBR v UH) }\end{array}$ & $\begin{array}{l}\text { ABN_ME_L9/ } \\
\text { ADJ_ME_L9 }\end{array}$ \\
\hline 10 & $\begin{array}{l}\text { Target(P_ABN/P_ADJ) } \Lambda \\
\text { Co_W(RB v JJS v JJR v } \\
\text { RBS v RBR v UH) } \Lambda \\
(\text { Auxiliary+not) } \\
\text { Target(N_INVB) } \Lambda \\
\text { Co_W(RB v JJS v JJR v } \\
\text { RBS v RBR v UH) }\end{array}$ & $\begin{array}{l}\text { ABN_ME_L10 } \\
/ \\
\text { ADJ_ME_L10 }\end{array}$ \\
\hline 11 & $\begin{array}{l}\text { Target(N_ABN/N_ADJ) } \Lambda \\
\text { Co_W(RB v JJS v JJR v } \\
\text { RBS v RBR v UH) } \Lambda \\
(\text { Modal+not }) \\
\text { Target(P_INVB) } \Lambda \\
\text { Co_W(RB v JJS v JJR v } \\
\text { RBS v RBR v UH) }\end{array}$ & $\begin{array}{l}\text { ABN_ME_L11 } \\
/ \\
\text { ADJ_ME_L11 }\end{array}$ \\
\hline 12 & $\begin{array}{l}\text { Target(N_ABN/N_ADJ) } \Lambda \\
\text { Co_W(RB v JJS v JJR v } \\
\text { RBS v RBR v UH) } \Lambda \\
(\text { Auxiliary+not) } \\
\text { Target(P_INVB) } \Lambda \\
\text { Co_W(RB v JJS v JJR v } \\
\text { RBS v RBR v UH) }\end{array}$ & $\begin{array}{l}\text { ABN_ME_L12 } \\
/ \\
\text { ADJ_ME_L12 }\end{array}$ \\
\hline
\end{tabular}

*L1 to L12 denotes the level1 to level12.

\subsection{Experiment setup}

The hyper parameters namely learning rate, batch size, epochs, dropout, word embedding were used in 
the proposed RCNN-GloVe model. Here, the GloVe word embedding dimensions were 300, two convolutional layers with the ReLu activation function, pooling layer was used for reducing the dimensionality on the features of filter to build the final feature with the two k-max pooling tasks. The dropout layer got included before the fully connected layer and the dropout rate for regularization was 0.5. The batch size of 10 with 10 epochs and a learning rate of 0.001 was used in CNN. Another proposed ML model of RSVM with GloVe was used as the comparative model for our research work. In this, the SVM-RBF classifier was used with the $C$ value of 1 and the gamma value of 0.0. 10-fold cross-validation

was used for training the data for both the RCNNGloVe and RSVM-GloVe models since it performed best. Sparse-categorical-cross entropy loss function was used for the loss function and the optimizer was adam.

\section{Result and discussion}

Classifying the tweet remains a difficult task in SA. Many researchers have tried for multi-class classification; however, it remains a perplexing task in most of the cases by throwing lot of challenges [29]. In reference to this research work classifying the tweets into multi-class classifications for complex sentences with mixed emotions, targeting the abstract nouns and adjective words do posed a considerable amount of challenging tasks. To resolve this problem, the proposed RCNN-GloVe and RSVM-GloVe were developed. To measure the effectiveness of our approach, the performance metrics Accuracy, Precision, Recall, F-measure were measured by using the below-mentioned equation:
- $\quad$ Precision $=$ True Positive $/$ (True Positive + False Positive)

- $\quad$ Recall $=$ True Positive / (True Positive + False Negative)

- F-Measure $=2 \times[($ Precision $\times$ Recall $) /$ $($ Precision + Recall $)]$

- $\quad$ Accuracy $=($ Number of correct predictions $/$ Total number of predictions)

Our proposed models have classified the tweet sentences into twelve classes (Levels) for abstract nouns and adjective sets of sentences separately which are as elaborated in section 3 . The accuracy of the classification is presented in Tables 4 and 5. From the results, it was observed that RCNN-GloVe got better results of accuracy, precision, recall and Fmeasure when compared with the other proposed rule-based modified RSVM-GloVe model when tested using the sentiment 140 twitter dataset.

In general, finding the emotions from the text is not a difficult task, which can either be positive emotions or negative emotions. But in the case of mixed emotions, there will be a combination of two opposite emotions at the same time [6]. Identifying the mixed emotions from the text data is a challenging one because of the presence of opposite valenced emotions, position of the negation words(such as no, not, never, etc.) and the presence of negative emotion words with the negation words. Our proposed rule-based models have overcome these challenges in identifying the mixed emotions from the twitter dataset and has achieved good results. Initially, the proposed fuzzy rules were applied to the RSVM classifier with word2vec, which resulted in very low levels of accuracy. This was because the word2vec model does not have any explicit global or

Table 4. The performance of the proposed RSVM-GloVe model for abstract and adjective set of mixed emotions from twitter data

\begin{tabular}{|l|l|l|l|l|l|l|l|l|}
\hline \multirow{2}{*}{$\begin{array}{l}\text { ME } \\
\text { Levels }\end{array}$} & $\begin{array}{l}\text { Rule based modified SVM for ABN set of } \\
\text { sentences }\end{array}$ & \multicolumn{2}{l}{ Rule based modified SVM for ADJ set of sentences } \\
\cline { 2 - 10 } & Acc & P & R & F-measure & Acc & P & R & F- measure \\
\hline L1 & $\mathbf{9 8 . 1 1}$ & 90.18 & 90.54 & 90.36 & $\mathbf{9 3 . 1 5}$ & 86.81 & 87.10 & 86.95 \\
\hline L2 & $\mathbf{9 7 . 9 2}$ & 91.16 & 89.94 & 90.54 & $\mathbf{8 7 . 2 4}$ & 87.83 & 87.47 & 87.65 \\
\hline L3 & $\mathbf{9 7 . 6 1}$ & 90.67 & 90.27 & 90.47 & $\mathbf{8 7 . 7 2}$ & 88.74 & 87.76 & 88.25 \\
\hline L4 & $\mathbf{9 0 . 6 7}$ & 85.00 & 88.40 & 86.67 & $\mathbf{8 9 . 7 3}$ & 81.39 & 84.68 & 83.00 \\
\hline L5 & 87.72 & 87.46 & 87.46 & 87.46 & 85.56 & 83.67 & 84.38 & 84.02 \\
\hline L6 & 85.61 & 89.13 & 86.84 & 87.97 & 85.61 & 85.84 & 86.64 & 86.24 \\
\hline L7 & 87.14 & 84.70 & 88.42 & 86.52 & 78.57 & 83.59 & 84.06 & 83.82 \\
\hline L8 & 71.43 & 85.58 & 83.47 & 84.51 & 74.86 & 83.63 & 82.52 & 83.07 \\
\hline L9 & 76.53 & 85.47 & 86.90 & 86.18 & 82.49 & 83.80 & 86.87 & 85.31 \\
\hline L10 & 88.01 & 86.33 & 85.62 & 85.98 & 85.06 & 80.08 & 85.32 & 82.62 \\
\hline L11 & 84.58 & 88.58 & 88.34 & 88.46 & 84.58 & 87.02 & 83.15 & 85.04 \\
\hline L12 & 89.55 & 89.73 & 87.42 & 88.56 & 85.68 & 88.41 & 80.37 & 84.20 \\
\hline Avg & $\mathbf{8 7 . 9 1}$ & 87.83 & 87.80 & $\mathbf{8 7 . 8 1}$ & $\mathbf{8 5 . 0 2}$ & 85.07 & 85.03 & $\mathbf{8 5 . 0 1}$ \\
\hline
\end{tabular}

International Journal of Intelligent Engineering and Systems, Vol.14, No.1, 2021

DOI: $10.22266 /$ ijies2021.0228.05 
Table 5. The performance of the proposed RCNN-GloVe model for abstract and adjective set of mixed emotions from twitter data

\begin{tabular}{|l|l|l|l|l|l|l|l|l|}
\hline \multirow{2}{*}{$\begin{array}{l}\text { ME } \\
\text { Levels }\end{array}$} & $\begin{array}{l}\text { Rule based modified CNN for ABN set of } \\
\text { sentences }\end{array}$ & \multicolumn{2}{l}{ Rule based modified CNN for ADJ set of sentences } \\
\cline { 2 - 10 } & Acc & P & R & F- measure & Acc & P & R & F- measure \\
\hline L1 & $\mathbf{9 9 . 4 3}$ & 93.14 & 92.43 & 92.79 & $\mathbf{9 8 . 1 1}$ & 90.80 & 91.04 & 90.92 \\
\hline L2 & $\mathbf{9 7 . 7 7}$ & 91.32 & 92.79 & 92.05 & $\mathbf{9 7 . 7 3}$ & 91.71 & 91.51 & 91.61 \\
\hline L3 & $\mathbf{9 9 . 3 3}$ & 92.53 & 94.24 & 93.38 & $\mathbf{9 9 . 3 3}$ & 90.64 & 91.85 & 91.24 \\
\hline L4 & $\mathbf{9 4 . 5 8}$ & 92.06 & 92.26 & 92.16 & $\mathbf{9 3 . 0 3}$ & 87.67 & 90.13 & 88.89 \\
\hline L5 & 93.15 & 94.42 & 91.36 & 92.87 & 94.13 & 89.53 & 90.39 & 89.96 \\
\hline L6 & 85.61 & 92.57 & 89.87 & 91.20 & 83.90 & 91.34 & 86.41 & 88.80 \\
\hline L7 & 87.14 & 91.75 & 90.90 & 91.32 & 88.85 & 86.37 & 89.04 & 87.68 \\
\hline L8 & 92.01 & 90.87 & 91.74 & 91.31 & 71.43 & 82.97 & 86.96 & 84.92 \\
\hline L9 & 90.24 & 90.28 & 92.76 & 91.50 & 76.53 & 84.98 & 89.16 & 87.02 \\
\hline L10 & 90.67 & 90.92 & 89.84 & 90.38 & 90.51 & 91.52 & 85.83 & 88.58 \\
\hline L11 & 84.58 & 91.18 & 92.35 & 91.76 & 84.46 & 88.57 & 85.17 & 86.83 \\
\hline L12 & 89.73 & 93.16 & 93.48 & 93.32 & 89.18 & 89.69 & 88.79 & 89.24 \\
\hline Avg & $\mathbf{9 2 . 0 2}$ & 92.02 & 92.00 & $\mathbf{9 2 . 0 0}$ & $\mathbf{8 8 . 9 3}$ & 88.82 & 88.86 & $\mathbf{8 8 . 8 1}$ \\
\hline
\end{tabular}

holistic information embedded in it by default. In our work, word embedding for abstract noun was performed using GloVe, which creates a global cooccurrence matrix by estimating the probability of a given word that will co-occur with other words. So, RSVM-GloVe produced better accuracy than the RSVM-Word2vec.

This led us to tune our RSVM parameters with the inclusion of GloVe in addition. This has resulted in a considerable increase in the overall accuracy level (87.91\% in abstract nouns and $85.02 \%$ in adjectives). Later, when the result of rule-based RSVM-GloVe model was compared with rule-based RCNN-GloVe model, it was found that the later model achieved fairly higher and better overall accuracy (92.02\% in abstract nouns and $88.93 \%$ in adjectives). Proposed RCNN-GloVe achieved an increased accuracy of $4.11 \%$ and $3.91 \%$ for abstract noun sentence classification and adjective sentence classification respectively when compared to our proposed RSVMGloVe model. The same trend was observed for other parameters like Precision, Recall and F- measure, where the proposed RCNN-GloVe model has distinctly outperformed the other proposed model of RSVM-GloVe. On the other hand, it was also observed that the accuracy rate in the first four levels of classification in both the proposed models got increased when compared to the other levels of classification (Level 5 to 12) this might be due to the factors like negation, misclassification.

Fig. 5 to Fig. 8 indicates the sparse-categoricalcross entropy loss function used in Keras for multiclass classification of RCNN-GloVe and RSVMGloVe models for abstract noun complex sentences and adjective complex sentences. While comparing the performance of the two models, it was found that our RCNN-GloVe model has outperformed over our RSVM-GloVe model.

Table 6 compares the performance of our best performed proposed model (RCNN-GloVe) with the recent existing works. It could be inferred from this that our proposed model has performed better by registering higher accuracy levels $(92.02 \%$ in abstract nouns and $88.93 \%$ in adjectives) when compared with the results from other studies. This could be mainly due to the use of fuzzy rule- based methods and multi-class classification along with CNN techniques. Similar findings were also made in another research that using of rule-based method along with CNN can improve the accuracy level [17]. On the other hand, the POS tag used in our research could not able to specifically differentiate between the abstract nouns and the other nouns in a correct way, which caused some misclassification, the same was also opined in other researches [17] as well. The use of optimized abstract noun algorithm in our proposed works could able to overcome this issue and enabled our proposed model to produce a better accuracy level.

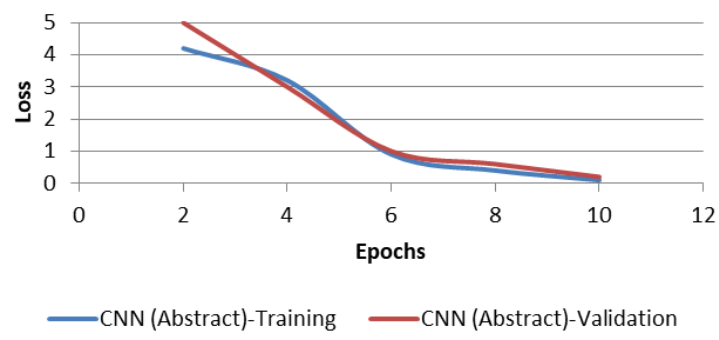

Figure. 5 RCNN-GloVe (abstract noun) loss 


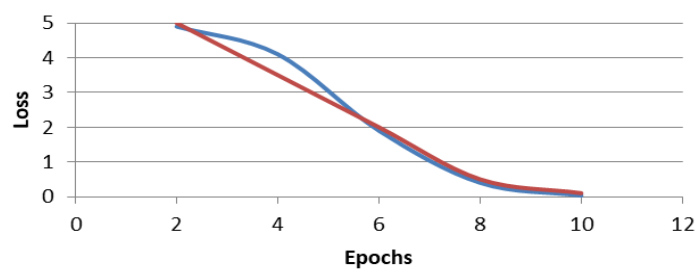

CNN (Adjective)-Training C CNN (Adjective)-Validation

Figure. 6 RCNN-GloVe (adjective) loss

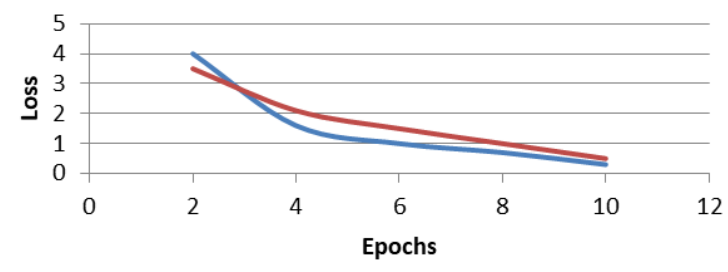

—SVM (Abstract)-Training _ SVM (Abstract)-Validation

Figure. 7 RSVM-GloVe (abstract noun) loss

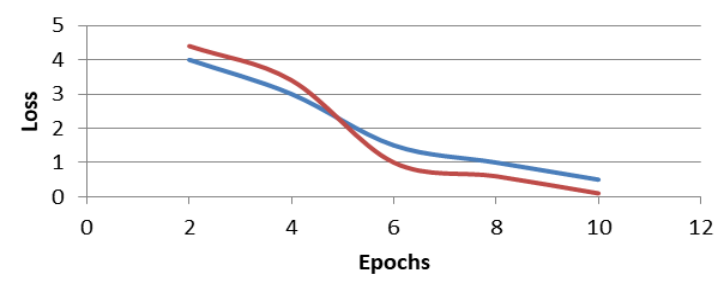

SVM (Adjective)-Training SVM (Adjective)-Validation

Figure. 8 RSVM-GloVe (adjective) loss

Table 6. Comparison of RCNN-GloVe with the other existing models on twitter dataset.

\begin{tabular}{|l|l|c|c|c|c|}
\hline $\begin{array}{l}\text { S.N } \\
0\end{array}$ & Journal Paper & $\begin{array}{l}\text { Rule } \\
\text { based } \\
\text { method }\end{array}$ & $\begin{array}{l}\text { Multi-class } \\
\text { classificatio } \\
\text { n }\end{array}$ & $\begin{array}{l}\text { CN } \\
\text { N }\end{array}$ & $\begin{array}{l}\text { Accuracy } \\
\%\end{array}$ \\
\hline 1 & {$[17]$} & $\checkmark$ & $\mathbf{x}$ & $\checkmark$ & 87.00 \\
\hline 2 & {$[27]$} & $\mathbf{x}$ & $\mathbf{x}$ & $\checkmark$ & 87.62 \\
\hline 3 & {$[29]$} & $\mathbf{x}$ & $\checkmark$ & $\mathbf{x}$ & 60.20 \\
\hline 4 & $\begin{array}{l}\text { RCNN- } \\
\text { GloVe } \\
\text { (Abstract } \\
\text { Noun) }\end{array}$ & $\checkmark$ & $\checkmark$ & $\checkmark$ & 92.02 \\
\hline 5 & $\begin{array}{l}\text { RCNN- } \\
\text { GloVe } \\
\text { (Adjective) }\end{array}$ & $\checkmark$ & $\checkmark$ & $\checkmark$ & 88.93 \\
\hline
\end{tabular}

\section{Conclusion}

Most of the existing works on SA in DL and ML techniques focused on binary and ternary classification. There are only limited works done so far by using multi-class classification. Hence our research focuses revolved around multi-class classification using DL and ML techniques namely, RCNN-GloVe and RSVM-GloVe for SA focusing on mixed emotions targeting abstract nouns and adjectives in twitter dataset. To perform this research, the three proposed algorithms were developed such as the proposed optimized complex sentences algorithm to extract all the complex sentences in a tweet precisely, optimized abstract noun algorithm to identify the abstract noun emotion words and adjective searching algorithm to retrieve all the sentences with adjectives. From our study, it is distinctly evident that the proposed rule-based CNN model with GloVe word embedding had an edge over the proposed SVM model with GloVe word embedding and also over other latest existing models with the highest accuracy level of $92.02 \%$ in abstract nouns and $88.93 \%$ in adjectives. In future works, these proposed models can be trained in various other domains like business research, advertising, medical management, etc. On the other hand, though this research has successfully demonstrated the multiclass classification of mixed emotions targeting abstract nouns and adjectives with fairly higher levels of accuracy, it could not able to quantify the emotions at different levels. Therefore, this has opened the doors to explore the possibilities of quantifying the emotions as a way forward for the forthcoming researches.

\section{Conflicts of Interest}

The authors declare that they have no conflicting interest that may be perceived as inappropriately influencing the representation or interpretation of reported research results of this research paper.

\section{Author Contributions}

The contributions of our research paper are as follows:

- Conceptualization: Christy Daniel and Loganathan Shyamala;

- Methodology: Christy Daniel;

- Software: Christy Daniel;

- Validation: Christy Daniel and Loganathan Shyamala;

- Formal analysis: Christy Daniel;

- Resources: Christy Daniel;

- Writing - original draft preparation: Christy Daniel;

- Writing - review and editing: Loganathan Shyamala;

- Supervision: Loganathan Shyamala. 


\section{Acknowledgments}

The authors wish to thank the School of Computer Science and Engineering, Vellore Institute of Technology, Chennai, India, for supporting us in this research.

\section{References}

[1] M. Madden, A. Lenhart, S. Cortesi, U. Gasser, M. Duggan, A. Smith, and M. Beaton, "Teens, Social Media, and Privacy", Pew Research Center, Washington, DC, pp. 21-86, 2013.

[2] A. Lenhart, M. Duggan, A. Perrin, R. Stepler, L. Rainie, and K. Parker, Teens, social media \& technology overview: Smartphones facilitate shifts in communication landscape for teens, Pew Research Center, Washington, DC, 2015.

[3] F. Abid, M. Alam, M. Yasir, and C. Li, "Sentiment Analysis Through Recurrent Variants Latterly on Convolutional Neural Network of Twitter", Future Generation Computer Systems, Vol. 95, pp. 292 - 308, 2019.

[4] B. Liu, "Text Sentiment Analysis Based on CBOW Model and Deep Learning in Big Data Environment", Journal of Ambient Intelligence and Humanized Computing, Vol. 11, pp. 451458, 2020.

[5] Statista, 2020, Number of monthly active Twitter users worldwide from 1st quarter 2010 to 1 st quarter 2019.

[6] J. T. Larsen and J. D. Green, "Evidence for Mixed Feelings of Happiness and Sadness from Brief Moments in Time", Cognition and Emotion, Vol. 27, No. 8, pp. 1469-1477, 2013.

[7] P. Williams and J. L. Aaker, "Can Mixed Emotions Peacefully Coexist?", Journal of Consumer Research, Oxford Academic, Vol. 28, No. 4, pp. 636-649, 2002.

[8] C. T. Fong, "The Effects of Emotional Ambivalence on Creativity", Academy of Management Journal, Vol. 49, No. 5, pp. 10161030, 2006.

[9] E. B. Andrade and J. B. Cohen, "On the Consumption of Negative Feelings", Journal of Consumer Research, Vol. 34, No. 3, pp. 283-300, 2007.

[10] S. H. Hemenover and U. Schimmack, "That's Disgusting, but Very Amusing: Mixed Feelings of Amusement and Disgust", Cognition and Emotion, Vol. 21, No. 5, pp. 1102-1113, 2007.

[11] C. C. Bee and R. Madrigal, "Consumer Uncertainty: the Influence of Anticipatory Emotions on Ambivalence, Attitudes, and
Intentions", Journal of Consumer Behaviour, Vol.12, No.5, pp.370-381, 2013.

[12] B. Liu, "Sentiment analysis: Mining Opinions, Sentiments, and Emotions", Cambridge University Press, 2015, ISBN 9781107017894.

[13] Your Dictionary, 2020, Examples of Abstract Nouns.

[14] A. Lenci, G. E. Lebani, L. C. Passaro, "The emotions of abstract words: A distributional semantic analysis", Topics in Cognitive Science, Vol. 10, No. 3, pp.550-572, 2018.

[15] J. K. Sing, S. Sarkar, and T. K. Mitra, "Development of a Novel Algorithm for Sentiment Analysis Based on AdverbAdjective-Noun Combinations", In: Proc. of 3rd National Conf. on Emerging Trends and Applications in Computer Science, Shillong, India, pp. 38-40, 2012.

[16] B. Pang, L. Lee, and S. Vaithyanathan, "Thumbs up?: Sentiment Classification Using Machine Learning Techniques", In: Proc. Conf. on Empirical Methods Natural Language Processing, Vol. 10, pp. 79-86, 2002.

[17] P. Ray and A. Chakrabarti, "A Mixed Approach of Deep Learning Method and Rule-Based Method to Improve Aspect Level Sentiment Analysis", Applied Computing and Informatics, 2019.

[18] V. Loia and S. Senatore, "A Fuzzy-Oriented Sentic Analysis to Capture the Human Emotion in Web-Based Content", Knowledge-Based Systems, Vol. 58, pp. 75-85, 2014.

[19] R. Plutchik, "The Nature of Emotions", American Scientist, Vol. 89, No .4, pp.344-350, 2001.

[20] N. F. Dasilva, E. R. Hruschka, and E. R. Hruschka Jr, "Tweet Sentiment Analysis with Classifier Ensembles", Decision Support Systems, Vol. 66, pp. 170-179, 2014.

[21] P. Katta and N. P. Hegde, "A Hybrid Adaptive Neuro-Fuzzy Interface and Support Vector Machine Based Sentiment Analysis on Political Twitter Data", International Journal of Intelligent Engineering and Systems, Vol. 12, No. 1, pp. 165-173, 2019.

[22] S. S. Maini and K. Govinda, "Stock Market Prediction using Data Mining Techniques", In: Proc. of IEEE International Conf. on Intelligent Sustainable Systems (ICISS), Tirupur, India, pp. 654-661, 2017.

[23] P. Soujanya, C. Erik, and G. Alexander, “Aspect Extraction for Opinion Mining with a Deep Convolutional Neural Network", KnowledgeBased Systems, Vol. 108, pp. 42-49, 2016. 
[24] A. Soufan, "Deep Learning for Sentiment Analysis of Arabic Text", In: Proc. of the ArabWIC 6th Annual International Conf. Research Track, Rabat, Morocco, pp. 1-8, 2019.

[25] S. Kiritchenko, X. Zhu, C. Cherry, and S. Mohammad, "NRC-Canada- 2014: Detecting Aspects and Sentiment in Customer reviews". In: Proc. of International Workshop on Semantic Evaluation, pp. 437- 442, 2014.

[26] A. Shoukry and A. Rafea, "A hybrid approach for Sentiment Classification of Egyptian Dialect Tweets", In: Proc. of IEEE First International Conf. on Arabic Computational Linguistics (ACLing), Cairo, Egypt, pp. 78-85, 2015.

[27] Z. Jianqiang, G. Xiaolin, and Z. Xuejun, "Deep Convolution Neural Networks for Twitter Sentiment Analysis", IEEE Access, Vol. 6, pp. 23253-23260, 2018.

[28] A. Hassan and A. Mahmood, "Convolutional Recurrent Deep Learning Model for Sentence Classification", IEEE Access, Vol.6, pp.39493957, 2018.

[29] M. Bouazizi and T. Ohtsuki, "A Pattern-Based Approach for Multi-Class Sentiment Analysis in Twitter", IEEE Access, Vol. 5, pp. 20617-20639, 2017.

[30] A. Go, R. Bhayani, and L. Huang, Twitter Sentiment Classification Using Distant Supervision, CS224N Project Report, Stanford, 2009.

[31] S. Bird and E. Loper, "NLTK: the Natural Language Toolkit", In: Proc. of the Association for Computational Linguistics on Interactive Poster and Demonstration Sessions, p. 31, 2004.

[32] http://help.sentiment140.com/for-students 